\title{
La diplomacia en la cumbre: el caso de APEC
}

DOI: $10.32870 /$ mycp.v4i13.647

\section{Jorge Arturo Smith Carbajal*}

$\mathbf{I}^{\mathrm{n}}$

ntroducción

Los cambios que ha sufrido el sistema internacional en las últimas tres décadas han originado, sin duda, cambios en los "modos" de hacer diplomacia, así como en el desarrollo de técnicas diplomáticas. En este sentido, se ha observado el incremento de un tipo de "diplomacia personal o directa" en la que participan jefes de Estado, para la cual se suele utilizar la expresión "diplomacia en la cumbre". Por medio de ella, los conductores de la política internacional establecen tratos de carácter personal y directo, con la finalidad de promover la imagen de su país y mejorar o iniciar algún tipo de relación, ya sea bilateral o multilateral. Un rasgo peculiar, es el contacto informal y sustancioso que se da entre los altos funcionarios, provocando un mayor acercamiento entre ellos, y por ende, entre los países que representan (al menos en teoría); además, cada uno funge como fuente de información primaria de la situación que prevalece en cada país.

Un aspecto importante de las cumbres, es su carácter público, que se da al congregar en una misma ocasión a líderes políticos. Al respecto, el papel de los medios de comunicación ha sido fundamental, debido a que han motivado la práctica de una diplomacia más abierta y pública que, en el sentido literal, se da "ante los ojos de todo el mundo". Además, como afirma Mario Amadeo "los pueblos tienen derecho a saber, sin duda, cuál es la índole de los compromisos contraídos por sus gobiernos y cuál es el alcance de sus obligaciones

* Estudiante del séptimo semestre de la licenciatura en Estudios Internacionales, de la Universidad de Guadalajara internacionales". De este modo se involucra a la opinión pública, a la par que se adquieren compromisos ante ella.

Otra característica es que los temas que se discuten, por el hecho de ser tratados por los jefes de Estado, suben de relieve. De esta manera, diversas problemáticas por las que atraviesa un país son puestas sobre la mesa de discusión, lo que facilita una mayor colaboración entre los países para llegar a soluciones más rápidas y eficaces.

Como ya se mencionó, el actual sistema internacional, inmerso en un inevitable proceso de globalización, ha dado lugar a crecientes niveles de interdependencia, como consecuencia de la integración de bloques económicos regionales (como es el caso de APEC), tornándose más común el uso de este tipo de diplomacia. La necesidad de que los jefes de Estado se reúnan se hace más evidente por la razón de que lo que ocurra en un país repercutirá inevitablemente en el resto del mundo.

Cabe señalar, que para el caso específico de México, el tema adquiere gran relevancia dado que será sede de los trabajos de APEC durante 2002. En este sentido, la presidencia de la república, ha incrementado en los últimos años sus actividades en torno a la región del Pacífico Asiático. Muestra de ello es la creciente participación de entidades gubernamentales adicionales a las tradicionales, que en ocasiones, durante momentos específicos de las negociaciones, llegan a adquirir un fuerte peso. Tal es el caso de la Secretaría de Economía, que en esta ocasión tendrá bajo su responsabilidad la organización de la cumbre de APEC en 2002. 
Para poder comprender y conocer mejor las ideas que hasta aquí he expresado, es necesario precisar algunos conceptos básicos sobre este "modo" de hacer diplomacia, conocido en inglés como summitry.

\section{Conceptos básicos}

En primer lugar, es conveniente contextualizar y dar cuenta de las características predominantes en las relaciones internacionales actuales. Algunos rasgos que definen a la diplomacia contemporánea son su carácter público, abierto, directo y más democrático, en el sentido que permite una mayor participación de los distintos actores del sistema internacional, a la vez que enriquece y 'redirecciona' la toma de decisiones y la evolución misma de las relaciones entre los Estados, y entre los Estados y otros actores (sean estos organismos internacionales, organizaciones no gubernamentales, empresas transnacionales, etc.). En síntesis, todo se conjuga en aras y como resultado de la democracia.

En segundo lugar, es preciso aclarar algunos detalles sobre la diferencia que existe entre cumbres y reuniones, debido a que con facilidad genera confusiones. Desde el punto de vista de la diplomacia, una cumbre es asistida exclusivamente por jefes de Estado, o líderes políticos de alto nivel, mientras que una reunión congrega a funcionarios políticos de menor nivel.

Otra particularidad consiste en que la función de las cumbres es simbólica, de representación (lo cual no evade la generación de efectos políticos y económicos) y se llevan a cabo periódicamente. En palabras del secretario de Relaciones Exteriores Dr. Jorge G. Castañeda "las cumbres son una rutina" 2 , entendida ésta, por su carácter periódico, por reunir prácticamente a los mismos países y por discutir temas en un margen de estabilidad, es decir, asuntos que no están sujetos a cambios constantes ni radicales, sino que forman parte ya de una agenda y que siguen la evolución natural y lógica de los acontecimientos. En las reuniones, por el contrario, es más factible que los asistentes (funcionarios políticos de menor nivel)hagan contribuciones serias e individuales en las negociaciones internacionales, lo cual se traduce de manera más inmediata en resultados tangibles o por lo menos visibles, debido en gran parte a las funciones de tipo operativo que desempeñan. En otras palabras, es en esta etapa de las reuniones, y a través de estos funcionarios, con quienes la celebración de acuerdos o los temas discutidos por los jefes de Estado comienzan a tomar forma; es ahí donde aquellos temas discutidos en las altas esferas de la política comienzan a enfrentarse con la realidad.

Conforme a la perspectiva de R.P. Barston, las cumbres además de tener un propósito simbólico, también sirven para obtener información, intercambiar puntos de vista, definir políticas estratégicas, resolver conflictos, así como para la negociación y ratificación de las etapas finales de los acuerdos, aspectos ya mencionados en párrafos anteriores.

Algunos especialistas en relaciones internacionales tienen consideraciones positivas respecto de las reuniones cumbre. Sin embargo, hay quienes enfatizan la escasa contribución que los jefes de Estado pueden realizar en las negociaciones internacionales por medio de las cumbres. Por ejemplo, se alude a que en ocasiones han llegado a realizar "concesiones imprudentes" con el fin de lograr negociaciones 
exitosas, además de que gran parte de las negociaciones están prácticamente concluidas mucho antes de que la cumbre se efectúe. Luis García Arias, especialista en la materia, repara sobre "tres puntos inconvenientes" de este tipo de diplomacia: en primer lugar se generan en la opinión pública ciertas expectativas difíciles de cumplir, que al no alcanzar los resultados esperados, provocan decepciones con un alto costo político; en segundo lugar, las diferencias ideológicas, psicológicas, culturales y de idioma pueden generar incomprensión y rivalidades entre los hombres de Estado y, finalmente, en ocasiones, las cumbres y las reuniones suelen carecer de la debida preparación técnica ${ }^{3}$. Este punto traería a colación dos aspectos: el primero, sobre el cuestionamiento de la práctica diplomática como resultado de la revolución de las comunicaciones y del contacto directo entre jefes de Estado ${ }^{4}$, que más que plantear su "extinción" estaría replanteando sus funciones, hoy en día más complejas e indispensables para la preparación técnica requerida en las cumbres y reuniones. El segundo punto confirma el anterior, por la necesidad de contar con toda una serie de preparativos técnicos de primer nivel a cargo de funcionarios diplomáticos, ya que, por ejemplo, se han presentado casos en que los gobernantes "se apartan de las líneas centrales de la política de sus países y deben, luego, desdecirse de sus promesas y compromisos" razón que plantea la necesidad de contar con la asesoría y desempeño técnico de expertos diplomáticos.

Lo anterior no es un patrón que se observe en todas las cumbres, más bien son apreciaciones de tipo general y por lo mismo a pesar de los inconvenientes o fallas que pueda representar este tipo de diplomacia, consideramos que son mayores los aspectos positivos que los inconvenientes. Prueba de ello son las cumbres de APEC, que aunque de manera lenta, han logrado avances y definiciones clave para el logro de sus objetivos. Por ejemplo, se han incorporado nuevos miembros, se ha intensificado la cooperación técnica y el nivel de relaciones económicas, y de manera indirecta se ha propiciado una mayor democratización en algunos países. A su vez, la democratización ha contribuido a mejorar la calidad y las condiciones en que se desarrollan los intercambios, no limitados exclusivamente al comercio.

Hay que añadir que la relevancia de las cumbres también se debe en gran parte al surgimiento y crecimiento de una diplomacia económica dentro del contexto del nuevo orden económico internacional, lo que ha llevado a interrelacionar los asuntos políticos y económicos. Por tal razón, los diplomáticos han tenido que incorporar, por un lado, como parte de sus actividades cotidianas, aquellas relacionadas con temas de índole comercial y económica, y por otro lado, a representantes de entidades de su gobierno, especializadas en esas ramas en las misiones de su país en el extranjero. Ejemplo de lo anterior es la embajada de México en Washington, D.C., que cuenta con representaciones de la Secretaría de Economía, de la Secretaría de Turismo y del Banco Mexicano de Comercio Exterior, entre otras, como resultado de la diversificación de las actividades de México en los Estados Unidos.

Un aspecto a considerar es el que se refiere a la institucionalización de las cumbres, en las que la diplomacia se torna un medio más eficaz para resolver las cuestiones que les da la razón de ser. En el caso de APEC, a pesar de ser un mecanismo que no ha madurado lo suficiente en su institucionalización (por el carácter de no obligatoriedad de algunos de sus planteamientos), la importancia de las cumbres es fundamental para reforzar lazos y ejercer presiones en ese sentido.

\section{Comentarios finales}

La diplomacia en la cumbre es un instrumento que el gobierno de México ha incorporado de 
manera activa en su política exterior. Los resultados que se puedan obtener en el ámbito internacional serán el reflejo de los avances al interior del país. Es decir, la proyección de México hacia el exterior, en el caso específico de APEC, mejorará en la medida en que las instancias gubernamentales que participan simultáneamente en cada una de estas cumbres, logren coordinarse adecuadamente, pero también de la incorporación de los puntos de vista de los diversos grupos de la sociedad, de manera que pueda lograrse un visión más completa sobre el papel que nuestro país desempeña y lo que espera lograr dentro de APEC, lo cual repercutirá sobre las posiciones estratégicas que se vayan definiendo a nivel internacional.

Tanto para la Cumbre de 2002, como para las posteriores, será fundamental resolver los siguientes problemas: a) la participación deficitaria que ha registrado México no sólo dentro de APEC, sino en otros foros, debido a la carencia de recursos financieros y humanos, principalmente en las Secretarías de Relaciones Exteriores y de Economía, y b) la promoción y aprovechamiento de los espacios ofrecidos por este foro, por parte del sector empresarial, para ampliar su panorama y poder adecuarse a las exigencias de innovación tecnológica, de manera que se adhieran a los estándares de productividad y competitividad en la región.

Actualmente -a pesar de que el proceso de integración en el Pacífico Asiàtico presenta una serie de obstáculos de carácter económico y político- no hay que olvidar que las economías de esta región han marchado a un ritmo dinámico, reafirmándose como una zona de grandes oportunidades económicas y políticas para México. En este sentido, hay conciencia de la enorme importancia que representa la cumbre de APEC para México en términos económicos; sin embargo no quiero dejar pasar la ocasión para reiterar y hacer hincapié sobre la gran oportunidad que nuestro país tiene para la diversificación de sus relaciones económicas, concentradas, en su mayor parte, con los Estados Unidos. No hay duda de que el gobierno ya está trabajando en la formulación de estrategias y políticas específicas, y en la mentalidad y adopción de una actitud encaminadas para tal fin, teniendo presente que toda relación internacional parte de una consolidación de lo nacional en los ámbitos de la economía, la política y la cultura.

\section{Notas}

1 Amadeo, Mario, Manual de politica internacional, Editorial Abeledo-Perrot.

2 Entrevista a Jorge G. Castañeda en el noticiario Imagen, realizada por Pedro Ferriz de Con el 23 de agosto de 2001.

3 Luis García Arias es retomado por Amadeo, Mario en op.cit.,capítulo VII.

4 De Icaza, Carlos (1999), La diplomacia contemporánea, Ed. Tercer Milenio, México, D.F.

5 Op. Cit. Amadeo, Mario.

\section{Fuentes}

Barston, R.P. (1997) Modern Diplomacy, $2^{\mathrm{a}}$ edición, Ed. Addison Wesley Longman; Campa, Homero, "En riesgo la cumbre de APEC" en PROCESO, sección economía, No.1284 del 10 de junio de 2001; Heater, Perek y Berridge, G.R., "Crises and Diplomacy" en Introduction to International Politics; Saner, Raymond, "Importancia del entrenamiento en gestión administrativa y liderazgo en la formación diplomática contemporánea", en Política Exterior Núm.60, SRE, junio 2000; De Icaza, Carlos (1999) La diplomacia contemporánea, Conaculta; Coleman, William y Underhill, Geoffrey (1998) Regionalism and Global Economic Integration. Europe, Asia and the Americas, First Published, Routledge, New York; González García, Juan José, La difícil integración de América Latina con el Pacífico Asiático, en Comercio Exterior (Bancomext), vol.48 No.1 ${ }^{9}$, noviembre de 1998. 